\title{
Convergence, Targeted Optimality, and Safety in Multiagent Learning
}

\begin{abstract}
This paper introduces a novel multiagent learning algorithm which achieves convergence, targeted optimality against memorybounded adversaries, and safety, in arbitrary repeated games. Called CMLeS, its most novel aspect is the manner in which it guarantees (in a PAC sense) targeted optimality against memory-bounded adversaries, via efficient exploration and exploitation. $C M L e S$ is fully implemented and we present empirical results demonstrating its effectiveness.
\end{abstract}

\section{Introduction}

In recent years, great strides have been made towards creating autonomous agents that can learn via interaction with their environment. When considering just an individual agent, it is often appropriate to model the world as being stationary, meaning that the same action from the same state will always yield the same (possibly stochastic) effects. However, in the presence of other independent agents, the environment is not stationary: an action's effects may depend on the actions of the other agents. This non-stationarity poses the primary challenge of multiagent learning (MAL) and comprises the main reason that it is best considered distinctly from single agent learning.

The simplest, and most often studied, MAL scenario is the stateless scenario in which agents repeatedly interact in the stylized setting of a matrix game (a.k.a. normal form game). In the multiagent literature, various criteria have been proposed to evaluate MAL algorithms, emphasizing what behavior they will converge to against various types of opponents, ${ }^{1}$ in such settings. The contribution of this paper is that it proposes a novel MAL algorithm, CMLeS, that for a

\footnotetext{
${ }^{1}$ Although we refer to other agents as opponents, we mean any agent (cooperative, adversarial, or neither)
}

Preliminary work. Under review by the International Conference on Machine Learning (ICML). Do not distribute. multi-player multi-action (arbitrary) repeated game, achieves the following three goals:

1. Convergence : converges to playing a Nash equilibrium in self-play (other agents are also $C M L e S$ );

2. Targeted Optimality : for any arbitrary $\epsilon>0$ and $\delta>0$, with probability at least $1-\delta$, achieves at least within $\epsilon+L(\delta)$ of the expected value of the best response against any memory-bounded, or adaptive, ${ }^{2}$ opponent of memory size $K$, in time polynomial in $\frac{1}{\epsilon}$, $\ln \left(\frac{1}{\delta}\right)$ and $\lambda^{-\operatorname{Size}(K+1)} . L(\delta) \in[0,1]$, is a decreasing function w.r.t. $1-\delta$ and assumes a very small value for small values of $\delta . \lambda$ is the minimum non-zero probability that the opponent assigns to an action, in any history and Size $(K+1)$ denotes number of feasible joint histories of size $K+1$. The same guarantee also holds for opponents which eventually become memorybounded, with the time complexity claim now holding from the point that the opponent becomes memorybounded. The main advance of $M L e S$ lies in reducing the exponential dependence on $\operatorname{Size}\left(K_{\max }\right)$ in time complexity, that is achieved by the current state of the art algorithm, to an exponential dependence on Size $(K+1)$, where $K_{\max }$ is an upper bound on the opponent's memory size, $K$.

3. Safety : converges to playing the maximin strategy against any other opponent which cannot be approximated as a $K_{\max }$ memory-bounded opponent.

\subsection{Related work}

Bowling et al. (Bowling \& Veloso, 2001) were the first to put forth a set of criterion for evaluating multiagent learning algorithms. In games with two players and two actions per player, their algorithm WoLF-IGA converges to playing best response against stationary, or memoryless, opponents (rationality), and converges to playing the Nash equilibrium in self-play (convergence). Subsequent approaches extended the rationality and convergence criteria to arbitrary (multi-player, multi-action) repeated games (Banerjee \& Peng, 2004; Conitzer \& Sandholm, 2006). Amongst them, AwESOME (Conitzer \& Sandholm, 2006) achieves conver-

\footnotetext{
${ }^{2}$ Consistent with the literature (Powers et al., 2005), we call memory-bounded opponents as adaptive opponents.
} 
gence and rationality in arbitrary repeated games without requiring agents to observe each others' mixed strategies. However, none of the above algorithms have any guarantee about the payoffs achieved when they face arbitrary non-stationary opponents. More recently, Powers et al. proposed a newer set of evaluation criteria that emphasizes compatibility, targeted optimality and safety (Powers \& Shoham, 2005). Compatibility is a stricter criterion than convergence as it requires the learner to converge within $\epsilon$ of the payoff achieved by a Pareto optimal Nash equilibrium. Their proposed algorithm, $\mathrm{PCM}(\mathrm{A})$ (Powers et al., 2007) is, to the best of our knowledge, the only known MAL algorithm to date that achieves compatibility, safety and targeted optimality against adaptive opponents in arbitrary repeated games.

\subsection{Contributions}

CMLeS improves on AwESOME by guaranteeing both safety and targeted optimality against adaptive opponents. It improves upon $\mathrm{PCM}(\mathrm{A})$ in five ways.

1. The only guarantees of optimality against adaptive opponents that $\mathrm{PCM}(\mathrm{A})$ provides are against the ones that are drawn from an initially chosen target set. In contrast, $C M L e S$ can model every adaptive opponent whose memory is bounded by $K_{\max }$. Thus it does not require a target set as input: its only input is $K_{\max }$, an upper bound on the memory size of adaptive opponents that it is willing to model and exploit.

2. $\mathrm{PCM}(\mathrm{A})$ achieves targeted optimality against adaptive opponents by requiring all feasible joint histories of size $K_{\max }$ to be visited a sufficient number of times. $K_{\max }$ for $\mathrm{PCM}(\mathrm{A})$ is the maximum memory size of any opponent from its target set. CMLeS significantly improves this by requiring a sufficient number of visits to all feasible joint histories only of size $K+1$. Thus $C M$ $L e S$ promises targeted optimality in number of steps polynomial in $\lambda^{- \text {Size }(K+1)}$ in comparison to $\operatorname{PCM}(\mathrm{A})$ which provides similar guarantees, but in steps polynomial in $\lambda^{-S i z e}\left(K_{\max }\right)$. The above sample efficiency property makes CMLeS a good candidate for online learning.

3. Unlike $\mathrm{PCM}(\mathrm{A}), C M L e S$ promises targeted optimality against opponents which eventually become memory-bounded with $K \leq K_{\max }$.

4. $\mathrm{PCM}(\mathrm{A})$ can only guarantee convergence to a payoff within $\epsilon$ of the desired Nash equilibrium payoff with a probability $\delta$. In contrast, CMLeS guarantees convergence in self-play with probability 1.

5. $C M L e S$ is relatively simple in its design. It tackles the entire problem of targeted optimality and safety by running an algorithm that implicitly achieves either of the two, without having to reason separately about adaptive and arbitrary opponents.

The remainder of the paper is organized as follows. Section 2 presents background and definitions, Section 3 and 4 presents our algorithm, Section 5 presents empirical results and Section 6 concludes.

\section{Background and Concepts}

This section reviews the definitions and concepts necessary for fully specifying $C M L e S$.

A matrix game is defined as an interaction between $n$ agents. Without loss of generality, we assume that the set of actions available to all the agents are same, i.e., $A_{1}=\ldots=A_{n}=A$. The payoff received by agent $i$ during each step of interaction is determined by a utility function over the agents' joint action, $u_{i}: A^{n} \mapsto \Re$. Without loss of generality, we assume that the payoffs are bounded in the range $[0,1]$. A repeated game is a setting in which the agents play the same matrix game repeatedly and infinitely often. A single stage Nash equilibrium is a stationary strategy profile $\left\{\pi_{i}^{*}, \ldots, \pi_{n}^{*}\right\}$ such that for every agent $i$ and for every other possible stationary strategy $\pi_{i}$, the following inequality holds: $E_{\left(\pi_{1}^{*}, \ldots, \pi_{i}^{*}, \ldots, \pi_{n}^{*}\right)} u_{i}(\cdot) \geq$ $E_{\left(\pi_{1}^{*}, \ldots, \pi_{i}, \ldots, \pi_{n}^{*}\right)} u_{i}(\cdot)$. It is a strategy profile in which no agent has an incentive to unilaterally deviate from its own share of the strategy. A maximin strategy for an agent is a strategy which maximizes its own minimum payoff. It is often called the safety strategy, because resorting to it guarantees the agent a minimum payoff.

An adaptive opponent strategy looks back at the most recent $K$ joint actions played in the current history of play to determine its next stochastic action profile. $K$ is referred to as the memory size of the opponent. ${ }^{3}$ The strategy of such an opponent is then a mapping, $\pi: A^{n K} \mapsto \Delta A$. If we consider opponents whose future behavior depends on the entire history, we lose the ability to (provably) learn anything about them in a single repeated game, since we see a given history only once. The concept of memory-boundedness limits the opponent's ability to condition on history, thereby giving us a chance to learning its policy.

We now specify what we mean by playing optimally against adaptive opponents. For notational clarity, we denote the other agents as a single agent $o$. It has been shown previously (Chakraborty \& Stone, 2008) that the dynamics of playing against such an $o$ can be modeled as a Markov Decision Process (MDP) whose transition probability function and reward function are

\footnotetext{
${ }^{3} K$ is the minimum memory size that fully characterizes the opponent strategy.
} 
determined by the opponents' (joint) strategy $\pi$. As the MDP is induced by an adversary, this setting is called an Adversary Induced MDP, or AIM in short.

An AIM is characterized by the $K$ of the opponent which induces it: the AIM's state space is the set of all feasible joint action sequences of length $K$. By way of example, consider the game of Roshambo or rockpaper-scissors (Figure 1) and assume that $o$ is a single agent and has $K=1$, meaning that it acts entirely based on the immediately previous joint action. Let the current state be $(R, P)$, meaning that on the previous action, $i$ selected $R$, and $o$ selected $P$. Assume that from that state, $o$ plays actions $R, P$ and $S$ with probability $0.25,0.25$, and 0.5 respectively. When $i$ chooses to take action $S$ in state $(R, P)$, the probabilities of transitioning to states $(S, R),(S, P)$ and $(S, S)$ are then $0.25,0.25$ and 0.5 respectively. Transitions to states that have a different action for $i, \operatorname{such}$ as $(R, R)$, have probability 0 . The reward obtained by $i$ when it transitions to state $(S, R)$ is -1 and so on.

The optimal policy of the MDP associated with the AIM is the optimal policy for playing against $o$. A policy that achieves an expected return within $\epsilon$ of the expected return achieved by the optimal policy is called an $\epsilon$-optimal policy (the corresponding return is called $\epsilon$-optimal return). If $\pi$ is known, then we can have computed the optimal policy (and hence $\epsilon$ optimal policy) by doing dynamic programming (Sutton \& Barto, 1998). However, we do not assume that $\pi$ or even $K$ are known in advance: they need to be learned in online play. We use the discounted payoff criterion in our computation of an $\epsilon$-optimal policy, with $\gamma$ denoting the discount factor.

Finally, it is important to note that there exist opponents in the literature which do not allow convergence to the optimal policy once a certain set of moves have been played. For example, the grim-trigger opponent in the well-known Prisoner's Dilemma $(P D)$ game, an opponent with memory size 1 , plays cooperate at first, but then plays defect forever once the other agent has played defect once. Thus, there is no way of detecting its strategy without defecting, after which it is impossible to recover to the optimal strategy of mutual cooperation. In our analysis, we constrain the class of adaptive opponents to include only those which do not negate the possibility of convergence to optimal exploitation, given any arbitrary initial sequence of exploratory moves (Powers \& Shoham, 2005).

Equipped with the required concepts, we are now ready to specify our algorithms. First, in Section 3, we present an algorithm that only guarantees safety and targeted optimality against adaptive opponents.

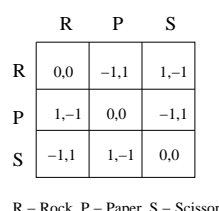

Roshambo

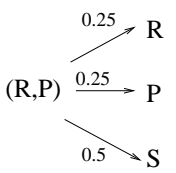

Opponent Strategy

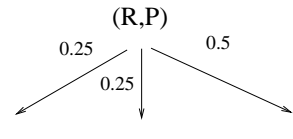

$(\mathrm{S}, \mathrm{R})$

$(\mathrm{S}, \mathrm{P})$

$(\mathrm{S}, \mathrm{S})$

Transition Function
Figure 1. Example of AIM

Then, in Section 4, we introduce the full-blown $C M L e S$ algorithm that incorporates convergence additionally.

\section{Model learning with Safety $(M L e S)$}

In this section, we introduce a novel algorithm, Model Learning with Safety (MLeS), that ensures safety and targeted optimality against adaptive opponents.

\subsection{Overview}

$M L e S$ begins with the hypothesis that the opponent is an adaptive opponent (denoted as o) with an unknown memory size, $K$, that is bounded above by a known value, $K_{\max }$. MLeS maintains a model for each possible value of $o$ 's memory size, from $k=0$ to $K_{\max }$, plus one additional model for memory size $K_{\max }+1$. Each model $\hat{\pi}_{k}$ is a mapping $A^{n k} \mapsto \Delta A$ representing a possible $o$ strategy. $\hat{\pi}_{k}$ is the maximum likelihood distribution based on the observed actions played by $o$ for each joint history of size $k$ encountered. Henceforth we will refer to a joint history of size $k$ as $s_{k}$ and the empirical distribution captured by $\hat{\pi}_{k}$ for $s_{k}$ as $\hat{\pi}_{k}\left(s_{k}\right)$. $\hat{\pi}_{k}\left(s_{k}, a_{o}\right)$ will denote the probability assigned to action $a_{o}$, by $\hat{\pi}_{k}\left(s_{k}\right)$. When a particular $s_{k}$ is encountered and the respective $o$ 's action in the next step is observed, the empirical distribution $\hat{\pi}_{k}\left(s_{k}\right)$ is updated. Such updates happen for every $\hat{\pi}_{k}$, on every step. For every $s_{k}, M L e S$ maintains a count value $v\left(s_{k}\right)$, which is the number of times $s_{k}$ has been encountered. We call an opponent model an $\epsilon$ approximation of $\pi$, when for any history of size $K$, it predicts the true opponent action distribution with error at most $\epsilon$.

On each step, $M L e S$ selects $\hat{\pi}_{\text {best }}$ (and correspondingly $k_{\text {best }}$ ) as the one from among the $K_{\max }+1$ (from 0 to $\left.K_{\max }\right)$ models that currently appears to best describe $o$ 's behavior. The mechanism for selecting $\hat{\pi}_{\text {best }}$ ensures that, with high probability, it is either $\hat{\pi}_{K}$ (the most compact representation of $\pi$ ) or a model with a smaller $k$ which is a good approximation of $\pi$. Once such a $\hat{\pi}_{\text {best }}$ is picked, MLeS takes a step towards learning an $\epsilon$-optimal policy for the underlying AIM induced by $k_{\text {best }}$. If it cannot determine such a $\hat{\pi}_{\text {best }}$, it defaults to playing the maximin strategy for safety.

Thus, the operations performed by $M L e S$ on each step 
can be summarized as follows:

1. Update all models based on the past step.

2. Determine $\hat{\pi}_{\text {best }}$ (and hence $k_{\text {best }}$ ). If a $\hat{\pi}_{\text {best }}$ cannot be determined, then return null.

3. If $\hat{\pi}_{\text {best }} \neq$ null, take a step towards solving the reinforcement learning (RL) problem for the AIM induced by $k_{\text {best }}$. Otherwise, play the maximin strategy.

Of these three steps, step 2 is by far the most complex. We present how MLeS addresses it next.

\subsection{Model selection}

The objective of $M L e S$ is to find a $k_{b e s t}$ which is either $K$ (the true memory size) or a suboptimal $k$ s.t. $\hat{\pi}_{k}$ is a good approximation of $\pi$ (o's true policy). It does so by comparing models of increasing size to determine at which point the larger models cease to become more predictive of o's behavior. We start by proposing a metric called $\Delta_{k}$, which is an estimate of how much models $\hat{\pi}_{k}$ and $\hat{\pi}_{k+1}$ differ from each other. But, first, we introduce two notations that will be instrumental in explaining the metric. We denote $\left(a_{i}, a_{o}\right) \cdot s_{k}$ to be a joint history of size $k+1$, that has $s_{k}$ as its last $k$ joint actions and $\left(a_{i}, a_{o}\right)$ as the last $k+1$ 'th joint action. For any $s_{k}$, we define a set $\operatorname{Aug}\left(s_{k}\right)=\cup_{\forall a_{i}, a_{o} \in A^{2}}\left(\left(a_{i}, a_{o}\right) \cdot s_{k} \mid v\left(\left(a_{i}, a_{o}\right) \cdot s_{k}\right)>0\right)$. In other words $\operatorname{Aug}\left(s_{k}\right)$ contains all joint histories of size $k+1$ which have $s_{k}$ as their last $k$ joint actions and have been visited at least once. $\Delta_{k}$ is then defined as $\max _{s_{k}, s_{k+1} \in A u g\left(s_{k}\right), a_{o} \in A} \mid \hat{\pi}_{k}\left(s_{k}, a_{o}\right)-$ $\hat{\pi}_{k+1}\left(s_{k+1}, a_{o}\right) \mid$. We say that $\hat{\pi}_{k}$ and $\hat{\pi}_{k+1}$ are $\Delta_{k}$ distant from one another.

Based, on the concept of $\Delta_{k}$, we make two observations that will come in handy for our theoretical claims made later in this subsection.

Observation 1. For all, $k \in\left[K, K_{\max }\right] \mid k \in$ $\mathbb{N}$, and for any $k$ sized joint history $s_{k}$ and any $s_{k+1} \in \operatorname{Aug}\left(s_{k}\right), E\left(\hat{\pi}_{k}\left(s_{k}\right)\right)=E\left(\hat{\pi}_{k+1}\left(s_{k+1}\right)\right)$. Hence $E\left(\Delta_{k}\right)=0$.

Let, $s_{K}$ be the last $K$ joint actions in $s_{k}$ and $s_{k+1}$. $\hat{\pi}_{k}\left(s_{k}\right)$ and $\hat{\pi}_{k+1}\left(s_{k+1}\right)$ represent draws from the same fixed distribution $\pi\left(s_{K}\right)$. So, their expectations will always be equal to $\pi\left(s_{K}\right)$. This is because $o$ just looks at the most recent $K$ joint actions in its history, to decide on its next step action.

Observation 2. For $k<K \mid k \in \mathbb{N}, \Delta_{k}$ is a random variable with $0 \leq E\left(\Delta_{k}\right) \leq 1$.

In this case, in the computation of $\hat{\pi}_{k}\left(s_{k}\right)$, the draws can come from different distributions. This is because, $k<K$ and there is no guarantee of stationarity of $\hat{\pi}_{k}\left(s_{k}\right)$. Thus, $\Delta_{k}$ can be any arbitrary random variable with an expected value within 0 and 1 .
High-level idea: Alg. 1 presents how $M L e S$ selects $k_{\text {best }}$. We denote the current values of $\hat{\pi}_{k}$ and $\Delta_{k}$ at time $t$, as $\hat{\pi}_{k}^{t}$ and $\Delta_{k}^{t}$ respectively.

Definition 1. $\left\{\sigma_{k}^{t}\right\}_{t \in 1,2, \ldots}$ is a sequence of real numbers, unique to each $k$, s.t. it satisfies the following:

1. it is a positive monotonically decreasing sequence, tending to 0 as $t \rightarrow \infty$;

2. for a fixed high probability $\rho>0$ and for $k \in$ $\left[K, K_{\max }\right], \operatorname{Pr}\left(\Delta_{k}^{t}<\sigma_{k}^{t}\right)>\rho$;

The reason for choosing such a $\left\{\sigma_{k}^{t}\right\}_{t \in 1,2, \ldots}$ sequence for each $k$ will be clear from the next two paragraphs. Later, we will show, how we compute the $\sigma_{k}^{t}$ 's. $M L e S$ iterates over values of $k$ starting from 0 to $K_{\max }$ and picks the minimum $k$ s.t for all $k \leq k^{\prime} \leq K_{\max }$, the condition $\Delta_{k^{\prime}}^{t}<\sigma_{k^{\prime}}^{t}$ is satisfied (steps 3-11).

For $k<K$, there is no guarantee that $\Delta_{k}$ will tend to 0 , as $t \rightarrow \infty$ (Observation 2). More often than not, $\Delta_{k}$ will tend to a positive value quickly. On the other hand, $\sigma_{k}^{t} \rightarrow 0$ as $t \rightarrow \infty$ (condition 1 of Definition 1 ). This leads to one of the following two cases:

1) $\sigma_{k}^{t}$ becomes $\leq \Delta_{k}^{t}$ and step 6 of Alg. 1 holds, thus rejecting $k$ as a possible candidate for selection.

2) $k$ gets selected. However, then we are sure that $\hat{\pi}_{k}^{t}$ is no more than $\sum_{k \leq k^{\prime}<K} \sigma_{k^{\prime}}^{t}$ distant from $\hat{\pi}_{K}^{t}$ (the best model of $\pi$ we have at present). With increasingly many time steps, $\hat{\pi}_{k}^{t}$ needs to be an increasingly better approximation of $\pi_{K}^{t}$, to keep getting selected.

For $k \geq K$, all $\Delta_{k}^{t}$ 's $\rightarrow 0$, as $t \rightarrow \infty$ (Observation 1 ). Since for all $k \geq K: \operatorname{Pr}\left(\Delta_{k}^{t}<\sigma_{k}^{t}\right)>\rho$ (condition 2 of Definition 1), $K$ gets selected with a high probability $\rho^{K_{\max }-K+1}$. A model with memory size more than $K$ is selected with probability at most $\left(1-\rho^{K_{\max }-K+1}\right)$, which is a small value.

We now address the final part of Alg. 1 that we have yet to specify: setting the $\sigma_{k}^{t}$ 's (step 2).

Choosing $\sigma_{k}^{t}$ : In the computation of $\Delta_{k}^{t}, M L e S$ chooses a specific $s_{k}^{t}$ from the set of all possible joint histories of size $k$, a specific $s_{k+1}^{t}$ from $\operatorname{Aug}\left(s_{k}^{t}\right)$ and an action $a_{o}^{t}$, for which the models $\hat{\pi}_{k}^{t}$ and $\hat{\pi}_{k+1}^{t}$ differ maximally on that particular time step. So,

$$
\Delta_{k}^{t}<\sigma_{k}^{t} \equiv\left|\hat{\pi}_{k}^{t}\left(s_{k}^{t}, a_{o}^{t}\right)-\hat{\pi}_{k+1}^{t}\left(s_{k+1}^{t}, a_{o}^{t}\right)\right|<\sigma_{k}^{t}
$$

The goal will be to select a value for $\sigma_{k}^{t}$ s.t. condition 2 of Definition 1 is always satisfied. Condition 1 will implicitly follow from the above. For $k \in\left[K, K_{\max }\right]$, we can rewrite Inequality 1 as,

$$
\begin{aligned}
\equiv \quad & \mid\left(\mid \hat{\pi}_{k}^{t}\left(s_{k}^{t}, a_{o}^{t}\right)-E\left(\hat{\pi}_{k}^{t}\left(s_{k}^{t}, a_{o}^{t}\right) \mid\right)-\left(\mid \hat{\pi}_{k+1}^{t}\left(s_{k+1}^{t}, a_{o}^{t}\right)\right.\right. \\
& -E\left(\hat{\pi}_{k+1}^{t}\left(s_{k+1}^{t}, a_{o}^{t}\right) \mid\right) \mid<\sigma_{k}^{t}
\end{aligned}
$$

The above step follows from using $E\left(\hat{\pi}_{k}^{t}\left(s_{k}^{t}, a_{o}^{t}\right)\right)=$ 


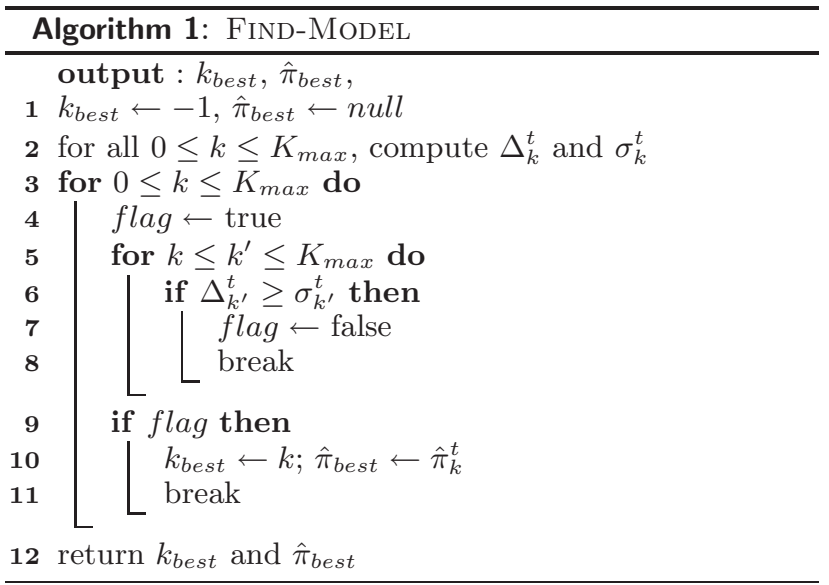

$E\left(\hat{\pi}_{k+1}^{t}\left(s_{k+1}^{t}, a_{o}^{t}\right)\right) \geq 0$ (Observation 1). One way to satisfy Inequality 2 is to have both $\left|\hat{\pi}_{k}^{t}\left(s_{k}^{t}, a_{o}^{t}\right)-E\left(\hat{\pi}_{k}^{t}\left(s_{k}^{t}, a_{o}^{t}\right)\right)\right|$ and $\mid \hat{\pi}_{k+1}^{t}\left(s_{k+1}^{t}, a_{o}^{t}\right)-$ $E\left(\hat{\pi}_{k+1}^{t}\left(s_{k+1}^{t}, a_{o}^{t}\right)\right) \mid$ be $<\sigma_{k}^{t}$. Thus, to ensure $[k \in$ $\left.\left[K, K_{\max }\right]: \operatorname{Pr}\left(\Delta_{k}^{t}<\sigma_{k}^{t}\right)>\rho\right]$, we need a lower bound of $\sqrt{\rho}$, on the probabilities of the above 2 inequalities.

Also, we observe that the following holds :

$\operatorname{Pr}\left(\left|\hat{\pi}_{k+1}^{t}\left(s_{k+1}^{t}, a_{o}^{t}\right)-E\left(\hat{\pi}_{k+1}^{t}\left(s_{k+1}^{t}, a_{o}^{t}\right)\right)\right|<\sigma_{k}^{t}\right)>\sqrt{\rho}$

$\Longrightarrow \operatorname{Pr}\left(\left|\hat{\pi}_{k}^{t}\left(s_{k}^{t}, a_{o}^{t}\right)-E\left(\hat{\pi}_{k}^{t}\left(s_{k}^{t}, a_{o}^{t}\right)\right)\right|<\sigma_{k}^{t}\right)>\sqrt{\rho}$

This can be derived by applying Hoeffding's inequality (Hoeffding, 1963) and using $v\left(s_{k}^{t}\right) \geq v\left(s_{k+1}^{t}\right)$. $v\left(s_{k}^{t}\right) \geq v\left(s_{k+1}^{t}\right)$ because the number of visits to a joint history $s_{k}$ must be at least the number of visits to any member from $\operatorname{Aug}\left(s_{k}\right)$. So,

$$
\begin{array}{r}
\operatorname{Pr}\left(\left|\hat{\pi}_{k+1}^{t}\left(s_{k+1}^{t}, a_{o}^{t}\right)-E\left(\hat{\pi}_{k+1}^{t}\left(s_{k+1}^{t}, a_{o}^{t}\right)\right)\right|<\sigma_{k}^{t}\right)>\sqrt{\rho} \\
\Longrightarrow \operatorname{Pr}\left(\Delta_{k}^{t}<\sigma_{k}^{t}\right)>\rho
\end{array}
$$

The problem now boils down to selecting a suitable $\sigma_{k}^{t}$ s.t. Inequality 5 is satisfied. Hoeffding's inequality gives us an upper bound for $\sigma_{k}^{t}$ in Inequality 5 . Using that upper bound and solving for $\sigma_{k}^{t}$, we get, $\sigma_{k}^{t}=\sqrt{\left(\frac{1}{2 v\left(s_{k+1}^{t}\right)} \ln \left(\frac{2}{1-\sqrt{\rho}}\right)\right)}$. So in general, for each $k \in\left[0, K_{\max }\right]$, the $\sigma_{k}^{t}$ value is set as above. Note that, $v\left(s_{k+1}^{t}\right)$ is the number of visits to the specific $s_{k+1}^{t}$ chosen for the computation of $\Delta_{k}^{t}$. Setting $\sigma_{k}^{t}$ as above satisfies both the conditions specified in Definition 1. Condition 1 follows implicitly since in infinite play, the action selection mechanism ensures infinite visits to all joint histories of a finite length.

Theoretical underpinnings: Now, we state our main theoretical result regarding model selection.

Lemma 3.1. After all feasible joint histories of size $K+1$ have been visited $\frac{(K+1)^{2}}{2 \epsilon^{2}} \ln \left(\frac{2}{1-\sqrt{\rho}}\right)$ times, then with probability at least $\rho^{K_{\max }+2}$, the $\hat{\pi}_{\text {best }}$ returned by Alg. 1 is an $\epsilon$ approximation of $\pi . \rho$ is the fixed high probability value from Condition 2 of Definition 1.

Proof. When all $k<K$ have been rejected, Alg. 1 selects $K$ with probability at least $\rho^{K_{\max }-K+1}$. If $p$ is the probability of selecting any $k<K$ as $k_{\text {best }}$, the probability of selecting any $k \leq K$ as $k_{\text {best }}$, is then at least $p+(1-p) \rho^{K_{\max }-K+1}>\rho^{K_{\max }-K+1}>\rho^{K_{\max }+1}$. If $k_{\text {best }}=K$, then we know that $\Delta_{K}^{t}<\sigma_{K}^{t}$. So from Inequality 4 ,

$$
\begin{aligned}
& \left.\operatorname{Pr}\left(\mid \hat{\pi}_{K}^{t}\left(s_{K}^{t}, a_{o}^{t}\right)-\pi\left(s_{K}^{t}, a_{o}^{t}\right)\right) \mid<\sigma_{K}^{t}\right)>\sqrt{\rho} \\
\Longrightarrow & \operatorname{Pr}\left(\left|\hat{\pi}_{K}^{t}\left(s_{K}^{t}, a_{o}^{t}\right)-\pi\left(s_{K}^{t}, a_{o}^{t}\right)\right|<\sigma_{K}^{t}\right)>\rho
\end{aligned}
$$

$s_{K}^{t}$ and $a_{o}^{t}$ are the respective joint history of size $K$ and action, for which models $\hat{\pi}_{K}^{t}$ and $\hat{\pi}_{K+1}^{t}$ maximally differ at $t$. So in this case, with probability $\rho, \hat{\pi}_{\text {best }}$ is a $\sigma_{K}^{t}$ approximation of $\pi$. In similar fashion it can be shown that if $k_{\text {best }}<K$, then with probability $\rho, \hat{\pi}_{\text {best }}$ is a $\sum_{k \leq k^{\prime} \leq K} \sigma_{k^{\prime}}^{t}$ approximation of $\pi$.

If an $\epsilon$ approximation of $\pi$ is desired, a sufficient condition is to ensure that for all $0 \leq k \leq K, \sigma_{k}^{t}$ gets assigned a value $\leq \frac{\epsilon}{K+1}$. If all feasible joint histo-

(3) ries of size $K+1$ are visited $\frac{(K+1)^{2}}{2 \epsilon^{2}} \ln \left(\frac{2}{1-\sqrt{\rho}}\right)$ times,

(4) then $\sigma_{K}^{t}$ must be less than $\frac{\epsilon}{K+1}$ (from Inequality 5 and Hoeffding's inequality). Also every feasible history of smaller sizes, must also have been visited at least $\frac{(K+1)^{2}}{2 \epsilon^{2}} \ln \left(\frac{2}{1-\sqrt{\rho}}\right)$ times. Hence $\sigma_{k}^{t}$ for all $k<K$ also must have values less than $\frac{\epsilon}{K+1}$.

For Alg. 1 to return an $\epsilon$ approximation of $\pi$, $M L e S$ does not need to know the number of visits (Lemma 3.1) required beforehand; it just needs to ensure that every feasible $K+1$ history gets visited so many times. Finally, what remains to be addressed is the action-selection mechanism (step 3, main algorithm).

\subsection{Action selection}

On each time step, the action selection mechanism decides on what action to take for the ensuing time step. If the $\hat{\pi}_{\text {best }}$ returned is null, it plays the maximin strategy. If $\hat{\pi}_{\text {best }} \neq$ null, the action selection strategy picks the AIM associated with opponent memory $k_{b e s t}$ and takes the next step in the reinforcement learning problem of computing a near-optimal policy for that AIM. In order to solve this RL problem, MLeS uses the variant of the R-Max algorithm that does not assume that the mixing time of the underlying MDP is known (Brafman \& Tennenholtz, 2003). R-Max is a model based RL algorithm that converges to playing an $\epsilon$-optimal policy for an MDP with probability 
$1-\delta$, in time complexity polynomial in $\frac{1}{\epsilon}, \ln \left(\frac{1}{\delta}\right)$ and certain parameters of the MDP. A separate instantiation of the R-Max algorithm is maintained for each of the possible $K_{\max }+1$ AIMs pertaining to the possible memory sizes of $o$, i.e, $\mathcal{M}_{0}, \mathcal{M}_{1}, \ldots, \mathcal{M}_{K_{\max }}$. On each step, based on the $k_{\text {best }}$ returned, the R-Max instance for the AIM $\mathcal{M}_{k_{\text {best }}}$ is selected to take an action.

The steps that ensure targeted optimality against adaptive opponents are then as follows:

1. First, ensure that Alg. 1 keeps returning a $k_{\text {best }} \leq$ $K$ with a high probability $\sqrt{1-\delta}$ s.t. $\hat{\pi}_{\text {best }}$ is an $\frac{\epsilon(1-\gamma)}{2 \operatorname{Size}(K)}$ approximation of $\pi$. The conditions for that to happen are given by Lemma 3.1. Playing optimally against such an approximation of $\pi$, guarantees an $\frac{\epsilon}{2}-$ optimal payoff against $o$ (Lemma 4 of (Brafman \& Tennenholtz, 2003)). Thus an $\frac{\epsilon}{2}$-optimal policy for such a model will guarantee an $\epsilon$-optimal payoff against $o$.

2. Once such a $k_{\text {best }} \leq K$ is selected by Alg. 1 with a high probability $\sqrt{1-\delta}$ on every step, then with a probability $\sqrt{1-\delta}$, converge to playing an $\frac{\epsilon}{2}$-optimal policy for $\mathcal{M}_{k_{\text {best }}}$. In order to achieve that, the R-Max instantiation for $\mathcal{M}_{k_{\text {best }}}$ will require a certain fixed number of visits to every joint history of size $k_{\text {best }}$. Since the $k_{\text {best }}$ selected by Alg. 1 is at most $K$ with a high probability, a sufficient number of visits to every joint history of size $K$ will suffice convergence to an $\frac{\epsilon}{2}$-optimal policy.

It can be shown that our R-Max-based action selection strategy implicitly achieves both of above steps in number of time steps polynomial in $\frac{1}{\epsilon}, \ln \left(\frac{1}{\delta}\right)$ and $\lambda^{- \text {Size }(K+1)}$. Note, we do not have the ability to take samples at will from different histories, but may need to follow a chain of different histories to get a sample pertaining to one history. In the worst case, the chain can be the full set of all histories, with each transition occurring with $\lambda$. Hence the unavoidable dependence on $\lambda^{-S i z e(K+1)}$, in time complexity. The bounds we provide are extremely pessimistic and likely to be tractable against most opponents. For example against opponents which only condition on $M L e S$ 's recent history of actions, $\lambda^{-\operatorname{Size}(K+1)}$ dependency gets replaced by a dependency over just $|A|^{K+1}$.

So far what we have shown is that $M L e S$, with a high probability $1-\delta$ on each step, converges to playing an $\epsilon$-optimal policy. It is important to note that, acting in this fashion does not guarantee it a return that is $1-\delta$ times the $\epsilon$-optimal return. However, we can compute an upper bound on the loss and show that the loss is extremely small for small values of $\delta$. Let $r_{t}$ be the random variable that denotes the reward obtained on time step $t$ by following the $\epsilon$-optimal policy. The maximum loss incurred is : $\mid(1-\delta) \sum_{t=0}^{\infty} \gamma^{t} E\left(r_{t}\right)-\sum_{t=0}^{\infty} \gamma^{t}(1-$ $\delta)^{t} E\left(r_{t}\right)|<| \sum_{t=0}^{\infty} \gamma^{t} E\left(r_{t}\right)-\sum_{t=0}^{\infty} \gamma^{t}(1-\delta)^{t} E\left(r_{t}\right) \mid \leq$ $\left|\sum_{t=0}^{\infty} \gamma^{t}-\sum_{t=0}^{\infty} \gamma^{t}(1-\delta)^{t}\right| \leq \frac{\gamma \delta}{(1-\gamma)(1-\gamma(1-\delta))}$. In the above computation, we assume that whenever $M L e S$ does not play the $\epsilon$-optimal policy, it gets the minimum reward of 0 . We denote this loss as $L(\delta)$, since it is a function of $\delta$ ( $\gamma$ being fixed). Note that $L(\delta)$ can be made extremely small by selecting a very small $\delta$.

This brings us to our main theorem regarding MLeS.

Theorem 3.2. For any arbitrary $\epsilon>0$ and $\delta>0$, $M L e S$ with probability at least $1-\delta$, achieves at least within $\epsilon+L(\delta)$ of the expected value of the best response against any adaptive opponent, in number of time steps polynomial in $\frac{1}{\epsilon}, \ln \left(\frac{1}{\delta}\right)$ and $\lambda^{-\operatorname{Size}(K+1)}$.

Against an arbitrary $o$, our claims rely on $o$ not behaving as a $K_{\max }$ adaptive opponent in the limit. This means $\Delta_{K_{\max }}$ tends to a positive value, as $t \rightarrow \infty$. Alg. 1 returns $\hat{\pi}_{\text {best }}$ as null in the limit, with probability 1. MLeS will then subsequently converge to playing the maximin strategy, thus ensuring safety.

\section{Convergence and Model learning with Safety ( $C M L e S$ )}

In this section we build on $M L e S$ to introduce a novel MAL algorithm for an arbitrary repeated game which achieves safety, targeted optimality, and convergence, as defined in Section 1. We call our algorithm, Convergence with Model Learning and Safety: (CM$L e S)$. CMLeS begins by testing the opponents to see if they are also running $C M L e S$ (self-play); when not, it uses $M L e S$ as a subroutine.

\subsection{Overview}

CMLeS (Alg. 2) can be tuned to converge to any Nash equilibrium of the repeated game in self-play. Here, for the sake of clarity, we present a variant which converges to the single stage Nash equilibrium. This equilibrium also has the advantage of being the easiest of all Nash equilibria to compute and hence has historically been the preferred solution concept in multiagent learning (Bowling \& Veloso, 2001; Conitzer \& Sandholm, 2006). The extension of CMLeS to allow for convergence to other Nash equilibria is straightforward, only requiring keeping track of the probability distribution for every conditional strategy present in the specification of the equilibrium.

Steps 1 - 2: Like Awesome, we assume that all agents have access to a Nash equilibrium solver and they compute the same Nash equilibrium profile. If there are finitely many equilibria, then this assumption can be lifted with each agent choosing randomly an equilibrium profile, so that there is a non-zero prob- 


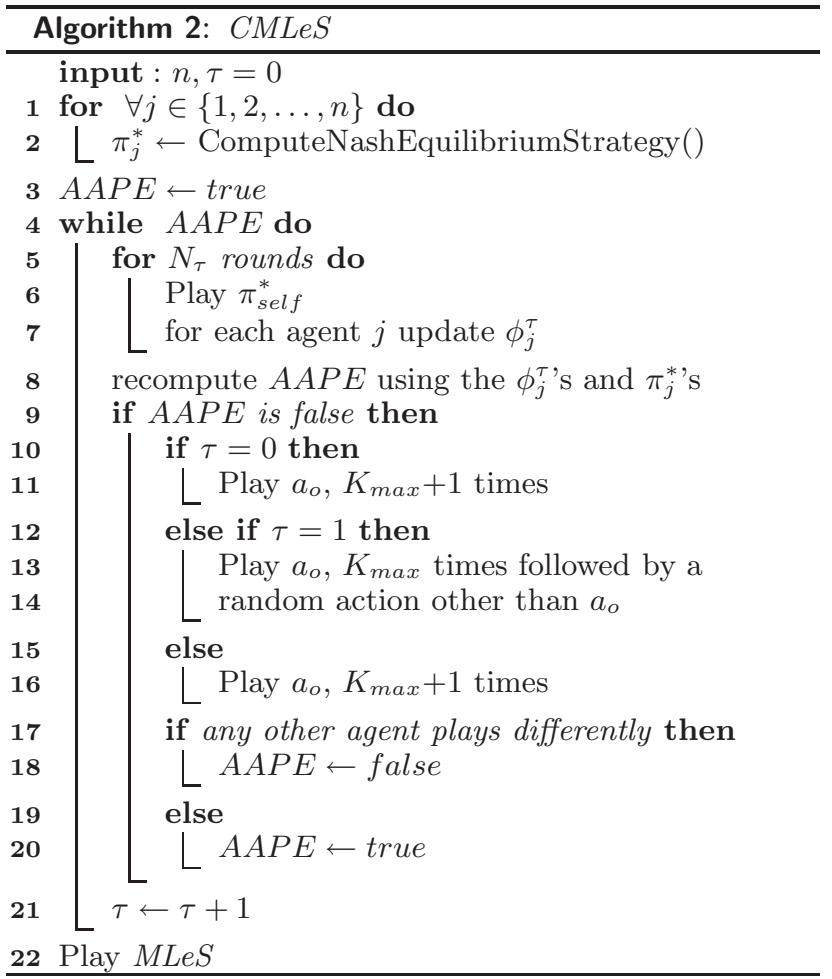

ability that the computed equilibrium coincides.

Steps 3 - 4: The algorithm maintains a null hypothesis that all agents are playing equilibrium $(A A P E)$. The hypothesis is not rejected unless the algorithm is certain with probability 1 that the other agents are not playing CMLeS. $\tau$ keeps count of the number of times the algorithm reaches step 4.

Steps 5 - 8 (Same as Awesome): Whenever the algorithm reaches step 5 , it plays the equilibrium strategy for a fixed number of episodes, $N_{\tau}$. It keeps a running estimate of the empirical distribution of actions played by all agents, including itself, during this run. At step 8 , if for any agent $j$, the empirical distribution $\phi_{j}^{\tau}$ differs from $\pi_{j}^{*}$ by at least $\epsilon_{e}^{\tau}$, AAPE is set to false. The $C M L e S$ agent has reason to believe that $j$ may not be playing the same algorithm. The $\epsilon_{e}^{\tau}$ and $N_{\tau}$ values for each $\tau$ are assigned in a similar fashion to AwESOME (Definition 4 of (Conitzer \& Sandholm, 2006)).

Steps 10 - 20: Once AAPE is set to false, the algorithm goes through a series of steps in which it checks whether the other agents are really CMLeS agents. The details are explained below when we describe the convergence properties of $C M L e S$ (Theorem 4.1).

Step 22: When the algorithm reaches here, it is sure (probability 1) that the other agents are not CMLeS agents. Hence it switches to playing $M L e S$.

\subsection{Theoretical underpinnings}

We now state our main convergence theorems.

Theorem 4.1. CMLeS satisfies both the criteria of targeted optimality and safety.

Proof. To prove the theorem, we need to prove:

1. For opponents not themselves playing $C M L e S, C M$ $L e S$ always reaches step 22 with some probability;

2. There exists a value of $\tau$, for and above which, the above probability is at least $\delta$.

Proof of 1 . We utilize the property that a $K$ adaptive opponent is also a $K_{\max }$ adaptive opponent (see Observation 1). The first time $A A P E$ is set to false, it selects a random action $a_{o}$ and then plays it $K_{\max }+1$ times in a row. The second time when $A A P E$ is set to false, it plays $a_{o}, K_{\max }$ times followed by a different action. If the other agents have behaved identically in both of the above situations, then $C M L e S$ knows : 1) either the rest of the agents are playing $C M L e S$, or, 2) they are adaptive and plays stochastically for a $K_{\max }$ bounded memory where all agents play $a_{o}$. The latter observation comes in handy below. Henceforth, whenever $A A P E$ is set to false, CMLeS always plays $a_{o}, K_{\max }+1$ times in a row. Since a non- $C M L e S$ opponent must be stochastic (from the above observation), at some point of time, it will play a different action on the $K_{\max }+1$ 'th step with a non-zero probability. $C M L e S$ then rejects the null hypothesis that all other agents are CMLeS agents and jumps to step 22 .

Proof of 2. This part of the proof follows from Hoeffding's inequality. $C M L e S$ reaches step 22 with a probability at least $\delta$ in $\tau$ polynomial in $\frac{1}{\kappa}$ and $\ln \left(\frac{1}{\delta}\right)$, where $\kappa$ is the maximum probability that any agent assigns to any action other than $a_{o}$ for a recent $K_{\max }$ joint history of all agents playing $a_{o}$.

Theorem 4.2. In self-play, CMLeS converges to playing the Nash equilibrium of the repeated game, with probability 1 .

The proof follows from the corresponding proof for Awesome (Theorem 3 of (Conitzer et al., 2006)).

\section{Results}

We now present empirical results that supplement the theoretical claims. We focus on how efficiently $C M L e S$ models adaptive opponents in comparison to existing algorithms, $\mathrm{PCM}(\mathrm{A})$ and Awesome. For CMLeS, we set $\epsilon=0.1, \delta=0.01$ and $K_{\max }=10$. To make the comparison fair with $\operatorname{PCM}(\mathrm{A})$, we use the same values of $\epsilon$ and $\delta$ and always include the respective opponent in the target set of $\mathrm{PCM}(\mathrm{A})$. We also add an adaptive strategy with $K=10$ to the target set of $\operatorname{PCM}(\mathrm{A})$, so that it needs to explore joint histories of size 10 . 
We use the 3-player Prisoner's Dilemma (PD) game as our representative matrix game. The game is a 3 player version of the N-player PD present in GAMUT. ${ }^{4}$ The adaptive opponent strategies we test against are : 1. Type 1: every other player plays defect if in the last 5 steps $C M L e S$ played defect even once. Otherwise, they play cooperate. The opponents are thus deterministic adaptive strategies with $K=5$.

2. Type 2: every other player behaves as type- 1 with 0.5 probability, or else plays completely randomly. In this case, the opponents are stochastic with $K=5$.

The total number of joint histories of size 10 in this case is $8^{10}$, which makes $\operatorname{PCM}(\mathrm{A})$ highly inefficient. However, $C M L e S$ quickly figures out the true $K$ and converges to optimal behavior in tractable number of steps. Figure 2 shows our results against these two types of opponents. The $\mathrm{Y}$-axis shows the payoff of each algorithm as a fraction of the optimal payoff achievable against the respective opponent. Also plotted in the same graph, is the fraction of times CMLeS chooses the right memory size (denoted as convg in the plot). Each plot has been averaged over 30 runs to increase robustness. Against type-1 opponents (Figure 2(i)), CMLeS figures out the true memory size in about 2000 steps and converges to playing optimally by 16000 episodes. Against type-2 opponents (Figure 2(ii)), it takes a little longer to figure out the correct memory size (about 35000 episodes) because in this case, the number of feasible joint histories of size 6 are much more. Both AwEsome and $\mathrm{PCM}(\mathrm{A})$ perform much worse. $\mathrm{PCM}(\mathrm{A})$ plays a random exploration strategy until it has visited every possible joint history of size $K_{\max }$, hence it keeps getting a constant payoff during this whole exploration phase.

Due to space constraints we skip the results for convergence and safety. However, it will be worthwhile to mention, that when $K_{\max }$ was set to $4, M L e S$ converged to playing the maximin strategy in about 10000 episodes, against both of the above opponents. The convergence part of $M L e S$ uses the framework of AwESOME and the results are exactly similar to it.

\section{Conclusion and Future Work}

In this paper, we introduced a novel MAL algorithm, $C M L e S$, which in an arbitrary repeated game, achieves convergence, targeted-optimality against adaptive opponents, and safety. One key contribution of CMLeS is in the manner it handles adaptive opponents: it requires only a loose upper bound on the opponent's memory size. In contrast, the existing state of the art algorithm, $\mathrm{PCM}(\mathrm{A})$, requires a complete specifica-

\footnotetext{
${ }^{4}$ http://gamut.stanford.edu/userdoc.pdf
}
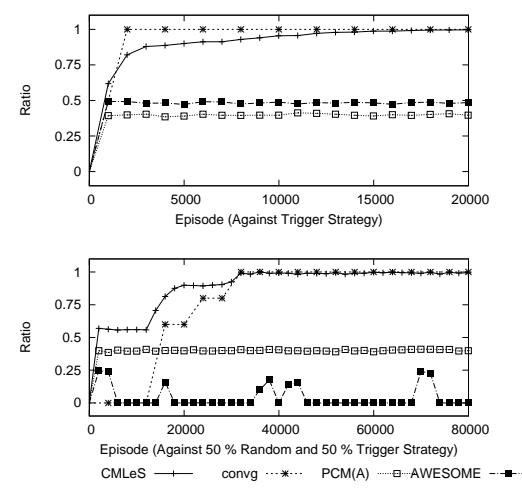

Figure 2. Against adaptive opponents

tion of the adaptive opponents at the beginning, which it calls a target set. Second, and more importantly, $C M L e S$ improves on $\mathrm{PCM}(\mathrm{A})$, by promising targeted optimality against adaptive opponents in time steps polynomial in $\lambda^{-\operatorname{Size}(K+1)}$ where Size $(K+1)$ is the number of feasible histories of size $K+1$, and $\lambda$ is the minimum non-zero probability that the opponent assigns to an action, in any history. $\mathrm{PCM}(\mathrm{A})$ guarantees the same, but in steps polynomial in $\lambda^{- \text {Size }\left(K_{\max }\right)}$.

Right now, the guarantees of $C M L e S$ are only in selfplay or when all other agents are adaptive. Any other distribution of agents is considered arbitrary, and $M L e S$ converges to playing the maximin strategy. Our ongoing research agenda includes improving $C M$ $L e S$ to have better performance guarantees against arbitrary mixes of agents, i.e., some adaptive, some selfplay, and the rest arbitrary.

\section{References}

Banerjee, B and Peng, J. Performance bounded reinforcement learning in strategic interactions. In $A A A I^{\prime} 04$

Bowling, $\mathrm{M}$ and Veloso, M. Convergence of gradient dynamics with a variable learning rate. In $I C M L$ '01

Brafman, R and Tennenholtz, M. R-max - a general polynomial time algorithm for near-optimal reinforcement learning. In J. Mach. Learn. Res.'03

Chakraborty, D and Stone, P. Online multiagent learning against memory bounded adversaries. In ECML'08

Conitzer, V and Sandholm, T. Awesome: A general multiagent learning algorithm that converges in self-play and learns a best response against stationary opponents. In J. Mach. Learn. Res.'06

Hoeffding, W. Probability inequalities for sums of bounded random variables. Journal of the American Statistical Association'1963

Powers, R and Shoham, Y. Learning against opponents with bounded memory. In IJCAI'05

Powers, R, Shoham, Y, and Vu, T. A general criterion and an algorithmic framework for learning in multi-agent systems. Mach. Learn.' 07

Sutton, $\mathrm{R}$ and Barto, A. Reinforcement Learning: An Introduction' 98 